\title{
Chromatic Derivative Filter Banks
}

\author{
M. J. Narasimha, Fellow, IEEE, A. Ignjatovic, and P. P. Vaidyanathan, Fellow, IEEE
}

\begin{abstract}
A new contribution to generalized sampling of bandlimited signals based on the so-called chromatic derivative operators was recently introduced by Ignjatovic. Chromatic derivatives are linear combinations of the ordinary derivatives, where the coefficients of the combination are derived from orthogonal polynomial theory. This letter describes the connection between these operators and the well-established ideas of perfect reconstruction and biorthogonality in analog filter banks.
\end{abstract}

Index Terms-Biorthogonality, Chebyshev-Bessel filter bank, chromatic derivatives, perfect reconstruction.

\section{INTRODUCTION}

I T IS WELL known that an analytic function can be expressed in terms of its derivatives using the classic Taylor series expansion. Although this series matches the function well in the neighborhood of expansion, the approximation error increases dramatically at far-away points when the infinite series is truncated. As such, the Taylor series expansion is not particularly useful in signal processing applications. For band-limited signals, however, it is possible to obtain perfect reconstruction (PR) of the signal with a finite number of derivatives evaluated at sub-Nyquist intervals, as shown in [3]. This generalized sampling scheme can be visualized as a filter bank in which the analysis filters evaluate the derivatives of the signal at uniform intervals and the synthesis filters perform the interpolation function. It is generally believed that the accurate evaluation of derivatives is a daunting task because of the impact of measurement noise. Even if their evaluation is not hampered by noise, this scheme is still not practical, since the frequency responses of the analysis filters that represent the higher order derivatives cluster together, which results in a poor encoding of the signal spectrum.

Chromatic derivatives were introduced in [1], [2] in order to alleviate the above-mentioned spectral encoding problem. They are related to standard derivatives in a manner similar to the way orthogonal polynomials are related to the monomials. The transfer functions of these derivatives are well separated in the frequency domain, and hence they encode the spectral information elegantly instead of destroying such information.

The main purpose of this letter is to demonstrate the PR property of filter banks based on chromatic derivatives. First, we derive the biorthogonality condition for PR in an infinite-channel

Manuscript received August 27, 2001; revised May 9, 2002. The associate editor coordinating the review of this manuscript and approving it for publication was Prof. Akbar Sayeed.

M. J. Narasimha is with the Department of Electrical Engineering, Stanford University, Stanford, CA 94305 USA (e-mail: sim@nova.stanford.edu).

A. Ignjatovic is with the School of Computer Science and Engineering, University of New South Wales, Sydney, NSW, Australia (e-mail: ignjat@cse.unsw.edu.au).

P. P. Vaidyanathan is with California Institute of Technology, Pasadena, CA 91125 USA (e-mail: ppvnath@systems.caltech.edu).

Publisher Item Identifier 10.1109/LSP.2002.801720.

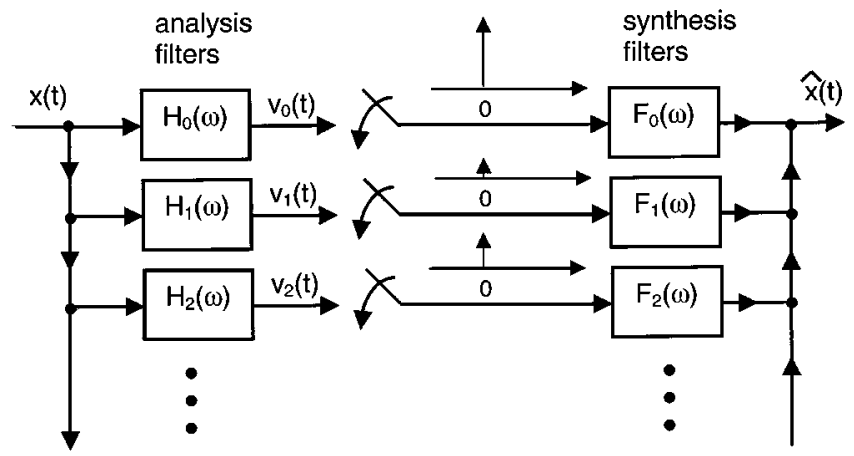

Fig. 1. The infinite-channel filter bank for a signal $x(t)$ band-limited to $-\pi<$ $\omega<\pi$.

continuous time filter bank (a review of standard results for discrete time PR filter banks can be found in [4]). This corresponds to the series expansion of a function, and we demonstrate that the Taylor filter bank satisfies this condition. Next, we introduce chromatic derivative filter banks using Chebyshev polynomials as an example and show that the corresponding interpolating functions for PR in such a filter bank are Bessel functions.

\section{Biorthogonal ANAlog Filter BANKS}

Consider Fig. 1, where a signal $x(t)$ band-limited to $|\omega|<\pi$ is passed through an infinite-channel analysis filter bank $H_{k}(\omega)$, whose outputs are sampled at $t=0$. Assume that the input $x(t)$ is in the space spanned by the synthesis filters $F_{k}(\omega)$, i.e., $x(t)=\sum_{m=0}^{\infty} c_{m} f_{m}(t)$, or equivalently $X(\omega)=\sum_{m=0}^{\infty} c_{m} F_{m}(\omega)$. If the analysis and synthesis filters satisfy the biorthogonality condition

$$
\int_{-\pi}^{\pi} H_{k}(\omega) F_{m}(\omega) \frac{d \omega}{2 \pi}=\delta_{k m}
$$

where $\delta_{k m}$ is the Kronecker delta function, then the filter bank has the PR property. To prove this, first consider the output of the $m$ th analysis filter evaluated at $t=0$ :

$$
\begin{aligned}
v_{m}(0) & =\int_{-\pi}^{\pi} X(\omega) H_{m}(\omega) \frac{d \omega}{2 \pi} \\
& =\sum_{k=0}^{\infty} c_{k} \int_{-\pi}^{\pi} F_{k}(\omega) H_{m}(\omega) \frac{d \omega}{2 \pi}=c_{m}
\end{aligned}
$$

where the last equality follows from the biorthogonality condition. The reconstructed signal is

$$
\hat{X}(\omega)=\sum_{m=0}^{\infty} v_{m}(0) F_{m}(\omega)=\sum_{m=0}^{\infty} c_{m} F_{m}(\omega)=X(\omega) .
$$

Of course, this is an "extreme filter bank," since there are infinitely many channels with only one sample kept in each subband. 
We can visualize the Taylor series expansion $x(t)=$ $\sum_{k=0}^{\infty} x^{(k)}(0) t^{k} / k$ ! of a signal $x(t)$ about $t=0$ as a filter bank of the form in Fig. 1. Since the $k$ th order differentiator has frequency response $(j \omega)^{k}$, the analysis filters can be written as $H_{k}(\omega)=(j \omega)^{k}, 0 \leq k \leq \infty$, and the impulse responses of the synthesis filters are $f_{m}(t)=t^{m} / m !, 0 \leq m \leq \infty$. The Fourier transform of $f_{m}(t)$ is $F_{m}(\omega)=2 \pi j^{m} \delta^{(m)}(\omega) / m$ !, where $\delta^{(m)}(\omega)$ is the $m$ th derivative of the Dirac delta function. It is easy to verify that the Taylor filter bank satisfies (1).

\section{Chromatic Derivative Filter Banks}

An interesting family of continuous-time filter banks, called chromatic derivative filter banks, arises when we define the $H_{k}(\omega)$ to be more general polynomials in $j \omega$ that are related to the classical orthogonal polynomials. In this case, the $k$ th analysis filter is a linear combination of derivatives of order less than $k$, and the coefficients of the combination are precisely the coefficients of the orthogonal polynomials, up to a sign. As an example of such a construction, consider a system where the $k$ th analysis filter $H_{k}(\omega)$ is derived from the $k$ th Chebyshev polynomial of the first kind $T_{k}(x)$. Recall that these polynomials are defined by the recursion

$$
T_{0}(x)=1, \quad T_{1}(x)=x, \quad T_{k+1}(x)=2 x T_{k}(x)-T_{k-1}(x) .
$$

Furthermore, these polynomials satisfy the orthogonality relation

$$
\int_{-1}^{1} W(x) T_{k}(x) T_{m}(x) d x=\delta_{k m} \gamma_{m}
$$

where the weighting function $W(x)$ and the normalization factor $\gamma_{m}$ are given by

$$
W(x)=\frac{1}{\sqrt{1-x^{2}}}, \quad \gamma_{m}= \begin{cases}\pi, & m=0 \\ \pi / 2, & m \neq 0 .\end{cases}
$$

Now, if we chose the analysis and synthesis filters as

$$
H_{k}(\omega)=j^{k} T_{k}(\omega / \pi)
$$

and

$$
F_{m}(\omega)=(-j)^{m}\left(2 / \gamma_{m}\right) W(\omega / \pi) T_{m}(\omega / \pi)
$$

then it can be verified, using (5), that the filter bank shown in Fig. 1 satisfies the biorthogonality condition (1). The factor $j^{k}$ in the definition of the analysis and synthesis filters guarantees that their impulse responses will be real.

It is noteworthy that the impulse responses of the synthesis filters in (7) can be expressed in terms of Bessel functions. The Fourier transform of the $m$ th-order Bessel function $J_{m}(\pi t)$ is band-limited to $|\omega|<\pi$, and its value in this range can be shown to be [5]

$$
(-j)^{m}(2 / \pi) T_{m}(\omega / \pi) W(\omega / \pi) .
$$

From (7) and (8), the impulse responses of the synthesis filters for PR can be written as

$$
f_{m}(t)= \begin{cases}J_{0}(\pi t), & m=0 \\ 2 J_{m}(\pi t), & m \neq 0\end{cases}
$$

It is also interesting to note that

$$
f_{m}(t)=(-1)^{m} 2 f_{0}(t) * h_{m}(t), \quad m \neq 0
$$

where $*$ denotes convolution. Hence, the $m$ th synthesis filter is the cascade of the base interpolation filter $f_{0}(t)=J_{0}(\pi t)$ and the $m$ th analysis filter $h_{m}(t)$, except for a gain term.

The analysis filters $H_{k}(\omega), 0 \leq k \leq \infty$, are known as chromatic derivative operators. Since these filters are derived from Chebyshev polynomials, they satisfy a recursive relationship similar to (4). The output $v_{k}(0)$ of the $k$ th analysis filter $H_{k}(\omega)$ in Fig. 1 is known as the $k$ th chromatic derivative of $x(t)$ evaluated at $t=0$. Denoting this by $K_{k}[x](0)$, we can write the following series expansion for $x(t)$ :

$$
x(t)=K_{0}[x](0) J_{0}(\pi t)+2 \sum_{n=1}^{\infty} K_{n}[x](0) J_{n}(\pi t) .
$$

Using (10), this expansion can also be written as

$$
\begin{aligned}
x(t)=K_{0}[x](0) J_{0}(\pi t) & \\
& +2 \sum_{n=1}^{\infty}(-1)^{n} K_{n}[x](0) K_{n}\left[f_{0}\right](t)
\end{aligned}
$$

where $K_{n}\left[f_{0}\right](t)$ denotes the $n$th chromatic derivative of the base interpolating function $f_{0}(t)$.

A comparison of the Taylor and the Chebyshev analysis banks reveals a number of useful differences. In the Taylor filter bank, all the filters have zeros at $\omega=0$, and the higher order filters are indistinguishable from each another. The Chebyshev analysis filters, on the other hand, have zeros distributed in the interval $-\pi<\omega<\pi$, and the zeros of any two analysis filters are disjoint. The filters are also well separated from each other.

\section{CONCLUSION}

Although we have described the Chebyshev-Bessel filter bank as an example, it is possible to formulate chromatic derivative filter banks corresponding to the other classical orthogonal polynomials. Since the use of an infinite number of filters is not practical, we propose to construct a local approximation to the signal about a point using a finite number of chromatic derivatives and truncate the interpolating functions appropriately. This process can then be repeated at a sequence of sampling points in order to obtain a piece-by-piece reconstruction of the signal. The procedure for putting the pieces together and an analysis of the resulting error characteristics are discussed in [1].

\section{REFERENCES}

[1] A. Ignjatovic. (2001, Feb.) Numerical differentiation and signal processing. Kromos Technology, Los Altos, CA. [Online] Tech. Rep. 1. Available: http://www.kromos.com.

[2] A. Ignjatovic and N. Carlin, "Method and a system of acquiring local signal behavior parameters for representing and processing a signal," U.S. Patent 6313 778, Nov. 2001.

[3] A. Papoulis, "Generalized sampling expansion," IEEE Trans. Circuits Syst., vol. CAS-24, pp. 652-654, Nov. 1977.

[4] P. P. Vaidyanathan, Multirate Systems and Filter Banks. Upper Saddle River, NJ: Prentice-Hall, 1993.

[5] A. Papoulis, Systems and Transforms With Applications in Optics. New York: McGraw Hill, 1968. 